\title{
Effets comparés du traitement à l'alcool des rattes gestantes et allaitantes sur les performances mnésiques de leurs progénitures
}

\author{
Prisca Joëlle Djoman DOUBRAN ${ }^{1 *}$, Koffi Mathias YAO $^{1}$ et Niemtiah OUATTARA ${ }^{2}$ \\ ${ }^{1}$ Laboratoire de Neurosciences, Université Félix HOUPHOUET-BOIGNY, 22 BP 582 Abidjan 22, \\ Côte d'Ivoire. \\ ${ }^{2}$ Departement of Neurology, Nanfang Hospital, Southern Medical University, Guangzou, Guangdong, Chine. \\ "Auteur correspondant ; E-mail : priscajo2002@yahoo.fr
}

\section{RESUME}

La consommation d'alcool par les mères gestantes ou allaitantes est un problème de santé publique qui a un impact chez la mère et le nourrisson. Les dommages causés chez le nouveau-né peuvent affecter la mémoire. L'objectif de cette étude est de comparer les effets de la prise d'alcool par des rattes durant les périodes de la gestation et/ou de la lactation sur les performances mnésiques des ratons. De ce fait, treize (13) lots de six (6) rattes nullipares sont accouplés avec des rats mâles à raison de deux femelles pour un mâle. Elles ont ensuite reçu quotidiennement de l'eau distillée (lot 0$)$ ou différentes doses d'alcool $(0,1 ; 0,3 ; 0,5$ et 0,8 g/L de sang) durant les périodes de gestation (groupes 1-4), de lactation (groupes 5-8) et du début de la gestation jusqu'à la fin de la lactation (groupes 9-12). Les performances mnésiques des ratons issus de chaque lot sont évaluées grâce à un labyrinthe en T. Les résultats ont montré que l'alcool a favorisé une baisse significative des pourcentages d'alternance spontanée des ratons $(\mathrm{P}<0,05)$. Cependant, les doses de $0,5 \mathrm{et} 0,8 \mathrm{~g} / \mathrm{L}$ de sang ont les plus fortes baisses avec 26 et $30 \%$ durant la gestation, et 10 et $8 \%$ pendant l'allaitement $(\mathrm{P}<0,0001)$. En conclusion, l'alcool a des effets délétères plus prononcés sur la mémoire lorsqu'il est consommé durant la période de la gestation.

(C) 2020 International Formulae Group. All rights reserved.

Mots clés : Performances mnésiques, Labyrinthe en T, Alternance spontanée.

\section{Comparative effects of alcohol treatment of pregnant and lactating rats on the memory performance of their offspring}

\begin{abstract}
Alcohol consumption by pregnant and lactating mothers is a public health problem that has an impact on mothers and newborn. Damage to the newborn can affect memory. The aim of this study is to compare the effects of alcohol consumption by rats during gestation and / or lactation on the memory performance of pups. Therefore, thirteen (13) lots of six (6) nulliparous rats are mated with male rats, two females for one male. They received daily distilled water (lot 0$)$ or different doses of alcohol $(0.1,0.3,0.5$ and $0.8 \mathrm{~g} / \mathrm{L}$ of blood) during gestation periods (groups 1 -4), lactation (groups 5-8) and from the start of gestation until the end of lactation (groups 9-12). The memory performance of each pup is evaluated using a T-maze. The results showed that the alcohol favored a significant drop in the percentages of spontaneous alternation of the pups $(\mathrm{P}<0.05)$. However, the doses of 0.5 and $0.8 \mathrm{~g} / \mathrm{L}$ of blood have the largest decreases with 26 and $30 \%$ during gestation,
\end{abstract}


and 10 and $8 \%$ during breastfeeding $(\mathrm{P}<0.0001)$. In conclusion, alcohol has more pronounced deleterious effects on memory when it is consumed during the gestation period.

(C) 2020 International Formulae Group. All rights reserved.

Keywords: Memory performance, T-maze, Spontaneous alternation

\section{INTRODUCTION}

L'alcool est une substance psychoactive dont les effets néfastes sur le Système Nerveux Central (SNC) peuvent varier selon le mode de consommation, la quantité consommée ou le stade de développement cérébral (De Ferron, 2015). Les effets délétères de cette substance ne s'exercent pas uniquement chez le consommateur, mais aussi indirectement chez le foetus et le nourrisson via le lait maternel. Dès la naissance, un nourrisson dont la mère a consommé de l'alcool durant la grossesse peut présenter des troubles du spectre de l'alcoolisation fotale (OMS, 2017). Les malformations du corps calleux associées à une anomalie neuro-développementale représentent la malformation cérébrale la plus fréquente à la naissance (Alby-Averseng, 2015).

La prévalence mondiale du Syndrome d'Alcoolisation Fœtal (SAF) reste élevée et probablement sous-estimée. De nombreuses études mettent en évidence une corrélation entre les doses d'alcool ingérées pendant la grossesse et la survenue du SAF sans pouvoir identifier une dose minimale sans risque fotal (Seror et al., 2009). Néanmoins, Doubran et al. (2018) ont montré que, toute consommation répétée d'alcool dosé à plus de $0,3 \mathrm{~g} / \mathrm{L}$ de sang par des rattes gestantes, exerce une activité potentiellement toxique sur la mémoire des ratons. La consommation de l'alcool pendant l'allaitement maternel est déconseillée en raison de la forte capacité de l'alcool consommée par une mère à atteindre les sécrétions maternelles telles que le lait (Beaulac-Baillargeon, 2008). Bien vrai que le risque d'affections induites chez le fotus et le nourrisson via le lait maternel soit réel, la consommation d'alcool par les mères gestantes et allaitantes est très courante dans nos sociétés.

L'objectif de cette étude est de comparer les effets de la prise de l'alcool pendant les périodes de gestation et/ou d'allaitement sur les aptitudes mnésiques des ratons dont les mères ont été traitées à différentes doses d'éthanol.

\section{MATERIEL ET METHODES \\ Matériel \\ Matériel animal}

Des rats de l'espèce Rattus norvegicus, de souche Wistar, sont utilisés pour la réalisation de cette étude. Ils proviennent tous $d u$ vivarium de l'Ecole Normale Supérieure (ENS, Abidjan, Côte d'Ivoire). Soixante-dixhuit (78) rattes nullipares non gravides âgées de 10 à 12 semaines et dont les poids varient entre 178 et $255 \mathrm{~g}$ sont reparties en 13 lots expérimentaux. Les rattes de chaque groupe expérimental sont accouplées avec 39 rats mâles en raison de deux femelles pour un mâle. Tous les animaux sans pathologie encéphalique connue étaient supposés sains. Ils étaient nourris et abreuvés ad libitum durant toute l'expérimentation. Les mâles ont servi à accoupler les femelles. Les femelles ont été traitées ou non avec une substance alcoolique (éthanol) pendant les périodes de gestation et/ou d'allaitement. Chaque ratte allaitante a allaité obligatoirement six (6) ratons selon (Koko et al., 2019). Les ratons sont utilisés pour la réalisation du test du labyrinthe en T.

\section{Matériel technique}

Le matériel technique est essentiellement composé d'un microscope optique, des lames et lamelles, d'une micropipette, d'une balance de précision, d'une sonde à gavage et d'un labyrinthe en $\mathrm{T}$. $\mathrm{Ce}$ dernier est construit en plexiglas de 
couleur grise. Le labyrinthe en $\mathrm{T}$ est conçu selon le modèle décrit par Badjo et al. (2017). Il comprend un compartiment de départ $(15 \times 10 \times 22)$, un couloir central $(45 \times 10 \times 22)$ et deux branches d'arrivée identiques (31x10x22). L'accès à chacun des compartiments peut être fermé grâce à un système de portes à guillotine à déplacement latéral.

\section{Méthodes}

\section{Procédure d'accouplement}

Les animaux sont repartis dans des cages par groupe de trois. Chaque groupe comporte deux femelles et un mâle. Les femelles sont examinées chaque matin afin de relever la présence de sperme ou de bouchon vaginal (Zougrou et al., 2019). Pour ce faire, un volume de $20 \mu \mathrm{L}$ de $\mathrm{NaCl} 0,9 \%$ a été prélevé à l'aide d'une micropipette et introduit dans le vagin de la ratte. Le mucus recueilli a été immédiatement sur une lame porte-objet, puis recouvert d'une lamelle avant l'observation au microscope optique (grossissement $X$ 400). Une fois la présence de spermatozoïdes déterminée chez les rattes, elles sont isolées et réparties dans des cages individuelles.

\section{Détermination des périodes de gestation et d'allaitement chez la ratte}

Le jour 0 de la gestation est défini comme le jour où l'on constate la présence de bouchon vaginal ou de sperme (OCDE 416, 2001). Quant à la période d'allaitement, elle débute chez la ratte après la mise basse et se poursuit $25 \pm 2$ jours (OCDE 426, 2007).

\section{Préparation de l'éthanol et détermination des quantités à administrer}

L'éthanol, titré à $96 \%$, a été dilué à $15 \%$. Les différents volumes d'alcool (15\%) à administrer aux rattes sont calculés selon la methode de Posey et Mozayani (2007).

$$
A=\frac{V \times P \times 0,8}{K \times m}
$$

A : éthanolémie approchée ;

$V$ : volume d'alcool ingéré en $\mathrm{ml}$;

$P$ : degré (\%) d'alcool de la boisson ingérée;

$K$ : coefficient de diffusion qui égale à 0,6

$m$ : masse de l'individu en $\mathrm{Kg}$;

0,8 : densité de l'éthanol.

\section{Formation des lots expérimentaux}

Pour la formation des groupes expérimentaux, les rattes sont reparties au hasard en treize (13) groupes de six (6) animaux chacun de manière à ce que les poids moyens de chaque groupe soient comparables entre eux (c'est-à-dire à $\pm 20 \%$ de la moyenne globale). Chaque ratte avait en sa possession six (6) ratons à allaiter. Les traitements per os à l'alcool sont effectués comme suit :

- Groupe $0:$ lot témoin traité uniquement à l'eau distillée du jour 0 de la gestation au dernier jour de la lactation

- Groupes $(1 ; 2 ; 3$ et 4$)$ : groupes traités du jour 0 de la gestation à la mise bas aux concentrations sanguines d'éthanol de 0,1 ; 0,$3 ; 0,5$ et $0,8 \mathrm{~g} / \mathrm{L}$.

- Groupes $(5 ; 6 ; 7$ et 8$)$ : groupes traités du jour 1 du post-partum au jour 23 de la lactation à des concentrations sanguines d'éthanol de 0,$1 ; 0,3 ; 0,5$ et $0,8 \mathrm{~g} / \mathrm{L}$.

- Groupes $(9 ; 10 ; 11$ et 12$)$ : groupes traités du jour 0 de la gestation au dernier jour 23 de la lactation à des concentrations sanguines d'éthanol de 0,$1 ; 0,3 ; 0,5$ et 0,8 $\mathrm{g} / \mathrm{L}$

\section{Sélection des ratons et réalisation du test de labyrinthe en $T$}

Tous les tests de mémoires des ratons ont été réalisés au premier jour de sevrage (Jour 24 du post- partum) (OCDE 426, 2007). Les ratons ont été regroupés en fonction des groupes de rattes formés ci-dessus. Pour le test du labyrinthe en $\mathrm{T}, 10$ ratons de chaque groupe expérimental, tirés au hasard sont admis au test de mémoire. Cependant, les individus malades et/ou chétifs (dont le poids corporel est inférieur de plus de deux écarts- 
types au poids moyen des petits de la même portée) ont été écartés.

\section{Test du labyrinthe en $T$ : Alternances spontanées sérielles}

Les alternances spontanées sont réalisées suivant la méthode de Deacon et Rawlins (2006) qui recommande une phase d'habituation, une session pré-test suivie du test.

\section{- Phase d'habituation}

Dans un premier temps, les animaux sont soumis à deux sessions d'habituation de 10 minutes (1 session par jour) au cours desquelles ils sont libres de visiter l'intégralité du labyrinthe. Cette phase permet aux ratons de se familiariser au labyrinthe en $\mathrm{T}$.

\section{- Session pré-test}

Les ratons sont soumis à une première session d'alternance séquentielle, ayant pour but d'une part, d'habituer les animaux à la procédure (confinement, ouverture/fermeture des portes, manipulation par l'expérimentateur), et d'autre part d'assurer la mise en place du comportement d'alternance puisque le fait de parcourir le labyrinthe permet à l'animal de réaliser une cartographie spatiale du labyrinthe. Cette session pré-test est composée de 5 essais consécutifs séparés par un intervalle inter-essai (IIE) de 30 secondes.

\section{- Session test}

Le jour suivant, les animaux sont soumis à la phase test. Cette session est composée de 20 essais consécutifs séparés par un IIE de 30 secondes. Au cours de chaque essai, l'animal est confiné dans le compartiment de départ pendant 30 secondes, puis la porte est ouverte et l'animal peut entrer librement dans l'un des deux bras d'arrivée du labyrinthe dans lequel il reste confiné durant un délai fixe de 30 secondes. Le raton est ensuite replacé dans le compartiment de départ pendant 30 secondes avant d'effectuer un nouvel essai.

\section{- Paramètres mesurés}

Chaque fois qu'un raton choisit d'entrer dans un bras d'arrivée différent de celui choisi précédemment, il alterne son choix et il lui est attribué un point. Dans le cas contraire, l'essai vaut 0 point. Le niveau d'alternance des animaux pour les 20 essais est calculé par le \% d'alternance :

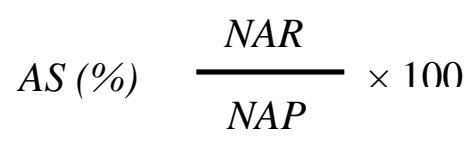

$\% A S$ : pourcentage d'alternance spontanée ; $N A R$ : nombre d'alternances réalisées ; NAP : nombre d'alternances possibles (19).

\section{Exploitation des résultats Analyse statistique}

Les valeurs sont exprimées sous forme de moyenne suivie de l'erreur standard sur la moyenne $(\mathrm{m} \pm \mathrm{esm})$. La signification des différences observées simultanément entre les lots traités et les lots témoins est appréciée en utilisant l'analyse des variances (ANOVA) du test de comparaison multiple de TukeyKramer via le logiciel de statistique GraphPad Prism 7 (Microsoft, San Diego California, USA). Dans ce test, lorsque :

- $P>0,05$ la différence observée est non significative (ns) ;

- $P<0,05$ la différence observée est peu significative (*);

- $P<0,01$ la différence observée est significative $(* *)$;

- $P<0,001$ la différence observée est très significative (***);

- $P<0,0001$ la différence observée est hautement significative $(* * * *)$.

\section{Représentations graphiques}

Les représentations graphiques des résultats sont réalisées grâce au logiciel GraphPad Prism 7 (Microsoft, San Diego California, USA).

\section{RESULTATS}

Effets du traitement à l'éthanol des rattes gestantes sur les performances mnésiques des ratons

Le taux d'alternance spontanée chez les ratons du lot témoin est estimé à $86,31 \pm$ 
$1,16 \%$. Ceux des ratons dont les mères sont traitées aux différentes doses d'éthanol (Eth) $(0,1 ; 0,3 ; 0,5$ et $0,8 \mathrm{~g} / \mathrm{L}$ de sang) sont respectivement évalués à $78,42 \pm 1,99 ; 75,42$ $\pm 2,1 ; 60,53 \pm 2,11$ et $56,84 \pm 2,05 \%$ (Tableau 1). En comparaison aux ratons du lot témoin, les doses Eth $(0,1 ; 0,3 ; 0,5$ et $0,8 \mathrm{~g} / \mathrm{L}$ de sang) ont diminué les performances mnésiques des ratons respectivement de 9,14 ; 12,$61 ; 29,86$ et $34,14 \%$. La comparaison statistique des effets enregistrés suite aux traitements alcooliques par rapport aux effets des ratons du lot témoin atteste que plus la quantité d'alcool administrée est élevée, plus la performance des ratons au test de performance mnésique est significativement faible $(\mathrm{p}<0,0001)$. En d'autres termes, la performance mnésique ou la capacité mnésique baisse avec l'alcool

\section{Effets du traitement à l'éthanol des rattes durant la lactation sur les performances mnésiques des ratons}

Les pourcentages d'alternance des ratons dont les mères sont traitées uniquement durant la lactation sont examinés. Les résultats montrent que chez les ratons dont les mères ont reçu les doses d'alcool de 0,$1 ; 0,3 ; 0,5$ et $0,8 \mathrm{~g} / \mathrm{L}$ de sang, les pourcentages d'alternance sont respectivement $80,48 \pm 4,07 ; 82,48 \pm$ 1,$68 ; 76,84 \pm 1,93$ et $78,94 \pm 1,26 \%$. Pour les ratons du lot témoin, la valeur enregistrée est de $86,31 \pm 1,16 \%$ (Figure 1). Comparativement au lot contrôle, les doses Eth $(0,1 ; 0,3 ; 0,5$ et $0,8 \mathrm{~g} / \mathrm{L}$ de sang $)$ ont respectivement diminué les performances mnésiques des ratons de 6,$75 ; 4,45 ; 10,97$ et $8,54 \%$. En référence au groupe contrôle, l'analyse statistique ré9vèle que toutes les doses d'alcool administrées ont induit une diminution des pourcentages d'alternance spontanée chez les ratons allaités. Les plus fortes baisses sont enregistrées aux doses de 0,5 et $0,8 \mathrm{~g} / \mathrm{L}$ de sang d'alcool $(\mathrm{p}<0,0001)$.
Effets du traitement à l'éthanol des rattes de la gestation à la lactation sur les performances mnésiques des ratons

Des rattes ont reçu un traitement éthanolique aux doses croissantes Eth $(0,1$; 0,$3 ; 0,5$ et $0,8 \mathrm{~g} / \mathrm{L}$ de sang) du début de la gestation jusqu'à la fin de la lactation. Les résultats de ce traitement sur les performances mnésiques relatives au test du labyrinthe en $\mathrm{T}$ sont respectivement $71,87 \pm 2,91 ; 68,50 \pm$ 3,$35 ; 50,00 \pm 5,26$ et $49,479 \pm 4,13 \%$ (Figure 2). En comparaison au lot témoin dont le pourcentage d'alternance spontanée est de $86,31 \pm 1,16 \%$, l'analyse statistique montre que tous effets enregistrés aux différentes doses d'alcool sont significativement en baisses par rapport au lot témoin ( $\mathrm{p}<0,0001)$. Relativement aux ratons du lot témoin, les baisses de performances mnésiques occasionnées par les doses Eth $(0,1 ; 0,3 ; 0,5$ et $0,8 \mathrm{~g} / \mathrm{L}$ de sang) sont respectivement estimées à 16,$73 ; 20,63 ; 42,06$ et $42,68 \%$.

\section{Incidence de la période de traitement des rattes à l'éthanol sur la performance mnésique des ratons}

Le traitement des rattes à la dose de 0,1 $\mathrm{g} / \mathrm{L}$ pendant la gestation et l'administration de la dose de $0,1 \mathrm{~g} / \mathrm{L}$ aux rattes pendant la lactation induisent des pourcentages d'alternance spontanée respectifs de 78,42 \pm $4,84$ et $80,48 \pm 4,07 \%$ ( $\mathrm{P}>0,05)$. Selon l'analyse statistique, il n'existe pas de différence significative entre ces valeurs. Par contre, lorsque les rattes sont traitées du début de la gestation jusqu'à la fin de la lactation, le pourcentage d'alternance spontanée des ratons $(71,87 \pm 2,91 \%)$ est significativement bas $(\mathrm{P}<$ 0,01) (Figure 3a).

S'agissant des différents traitements à Eth $(0,3 ; 0,5$ et $0,8 \mathrm{~g} / \mathrm{L}$ de sang), il est constaté une baisse significative des performances des ratons lorsque les rattes sont traitées uniquement durant la gestation et du début de la gestation jusqu'à la fin de la lactation. Cependant, la plus forte baisse est enregistrée durant la période de traitement gestation + lactation $(\mathrm{P}<0,01)$ (Figures 3b, c, d). 
Tableau I : Incidence du traitement des rattes à l'éthanol pendant la gestation sur la performance mnésique des ratons.

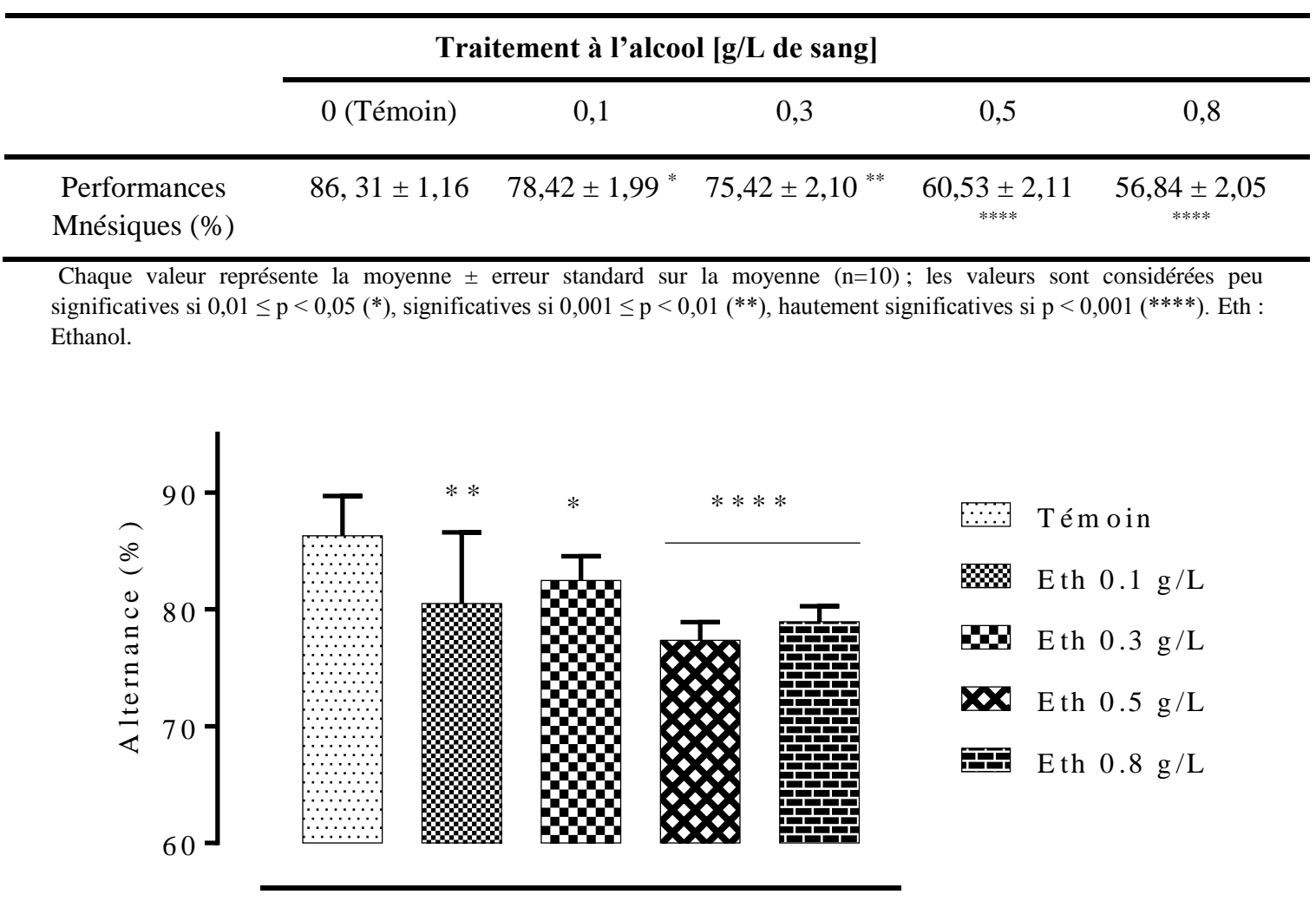

Figure 1 : Incidence du traitement des rattes à l'éthanol pendant la lactation sur la performance mnésique des ratons. Chaque valeur représente la moyenne \pm erreur standard sur la moyenne $(\mathrm{n}=10)$; les valeurs sont considérées peu significatives si $0,01 \leq \mathrm{p}<0,05\left(^{*}\right)$, significatives si $0,001 \leq \mathrm{p}<0,01\left(^{* *}\right)$; hautement significatives si $\mathrm{p}<$ $0,001(* * * *)$. Eth : Ethanol.
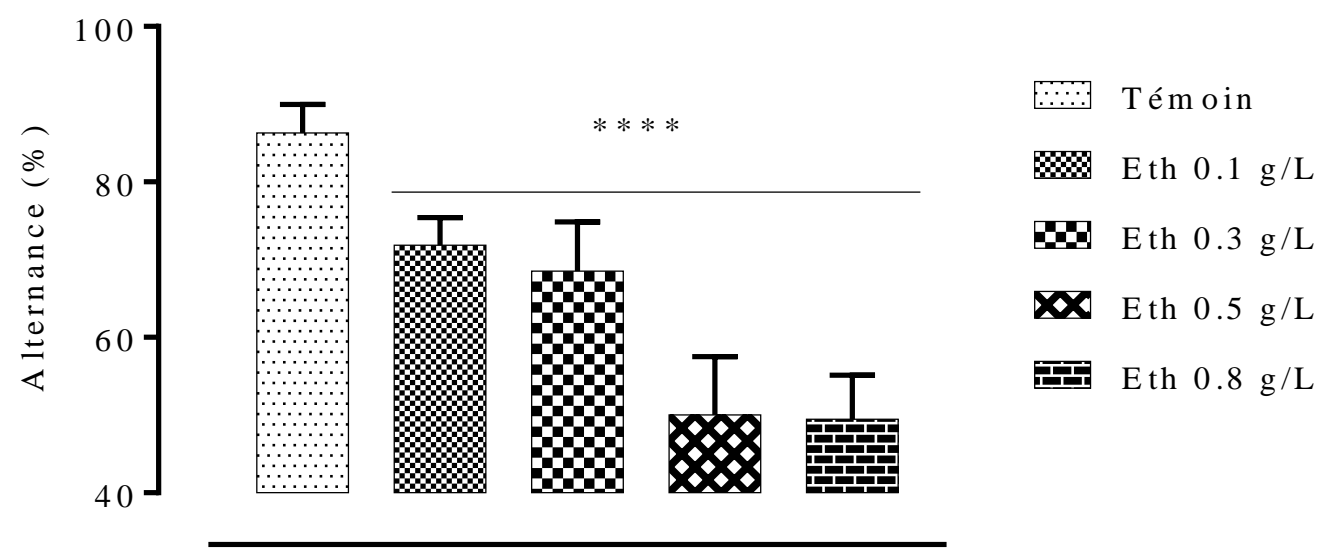

Figure 2: Incidence du traitement des rattes à l'éthanol pendant la gestation+lactation sur la performance mnésique des ratons. Chaque valeur représente la moyenne \pm erreur standard sur la moyenne $(n=10)$; les valeurs sont hautement significatives si p $<0,0001(* * * *)$; Eth : Ethanol. 


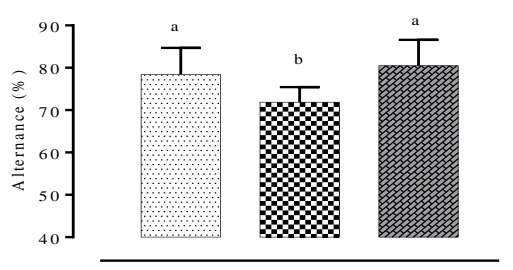

$\mathbf{a}$

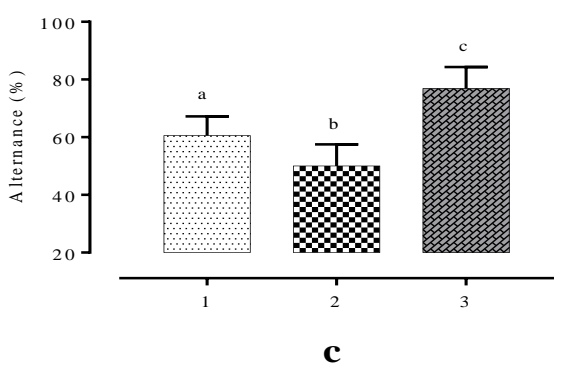

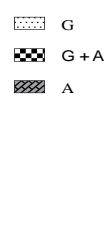

(1)

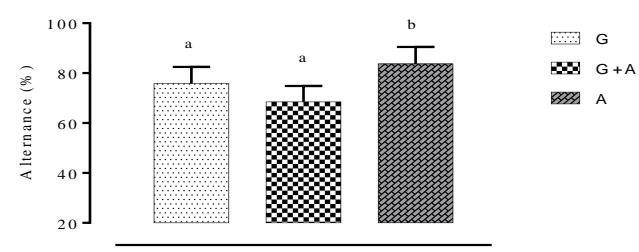

$\mathbf{b}$
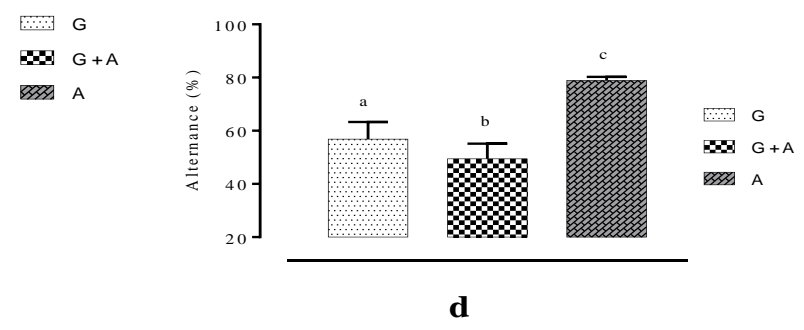

Figure 3 : Effets comparés du traitement des rattes à Ethanol pendant les périodes de la gestation et de la lactation sur les performances mnésiques des ratons.

Chaque valeur représente la moyenne \pm Ecart-type $(n=10)$, Aucune différence significative n'est observée entre les bandes surmontées de la même lettre. Fig $3 \mathrm{a}$ : traitement à la dose de Eth $(0,1 \mathrm{~g} / \mathrm{L}$ de sang); Fig $3 \mathrm{~b}:$ traitement à la dose de Eth $(0,3$ $\mathrm{g} / \mathrm{L}$ de sang); Fig 3c : traitement à la dose de Eth $(0,5 \mathrm{~g} / \mathrm{L}$ de sang $)$; Fig $3 \mathrm{~d}:$ traitement à la dose de Eth $(0,8 \mathrm{~g} / \mathrm{L}$ de sang) ; G : période de gestation, G+A : périodes de gestation et d'allaitement ; A : période d'allaitement.

\section{DISCUSSION}

Le test d'alternance spontanée dans le labyrinthe en $T$ permet d'évaluer les capacités cognitives des ratons dont les mères ont été soumises à une alcoolisation journalière (Deacon et al., 2006). Les ratons dont les mères sont traitées quotidiennement aux doses modérées d'alcool pendant la période de gestation et celle de la lactation réalisent des taux d'alternance significativement bas en comparaison aux ratons du lot témoin. Selon Bouamra et al. (2016), un déclin de la mémoire épisodique est lié à l'âge chez des rats à travers un test au labyrinthe en Y. La faible performance enregistrée chez des ratons, relate le déficit intellectuel causé chez un nourrisson par la consommation d'alcool de sa mère durant la grossesse (Du Plooy et al., 2016). L'alcool est une substance psychoactive ayant une très grande facilité de passage du sang maternel vers le sang fotal à travers le placenta. Une fois dans le sang fotal, il induit une neurodégénérescence par blocage des récepteurs N-méthyl-D-aspartate (NMDA)glutamate et par l'activation des récepteurs de l'Acide Gamma Aminobutyrique (GABA) (Ikonomidou, 2000).

En outre, une alcoolisation journalière pendant la période «gestation + allaitement » réduit significativement le taux d'alternance des ratons en comparaison aux effets enregistrés lorsque les rattes sont traitées uniquement durant la période de la gestation. Cette constatation démontre que le lait maternel constitue l'une des voies privilégiées de l'alcool pour atteindre le sang d'un enfant allaite. Selon Giglia et al. (2016), une consommation de l'alcool par la mère allaitante pourrait altérer les aptitudes intellectuelles et le comportement du nourrisson à l'âge adulte. 
L'analyse des effets de l'alcoolisation maternelle sur la mémoire des ratons illustre que les effets inhibiteurs de la performance mnésique de l'alcool sur le fotus sont plus importants que ceux induits chez le nourrisson via le lait maternel. Néanmoins, les concentrations d'alcool dans le lait maternel ressemblent étroitement à celles du sang maternel (Mennella et Beauchamp, 1991). Par conséquent, les effets moindres enregistrés durant l'allaitement en comparaison à la période de gestation, s'explique par la capacité des organes épurateurs (reins, foie) des ratons allaités à métaboliser l'alcool bien vrai qu'ils soient immatures. Cependant, ces organes pourrontils éliminer l'alcool sans pour autant subir de dommages histologiques?

De minimes changements de comportement chez les nourrissons exposés au lait contenant de l'alcool ont été signalés. Les autres conséquences à long terme pour les enfants de mères consommatrices d'alcool n'ont pas été démontrées de manière convaincante qu'elles affectent négativement les nourrissons allaités (Haastrup et al., 2014). Par ailleurs, la conclusion des travaux de Cebolla et al. (2009) montre que le dysfonctionnement cérébelleux induit par la consommation maternelle d'éthanol chez le raton dépend du régime d'alcool pendant la grossesse et non pendant la lactation.

\section{Conclusion}

L'alcool a des effets délétères sur les aptitudes mnésiques d'un foetus et d'un nourrisson allaité via le lait maternel. En comparaison à la période de la lactation, l'effet délétère sur la performance mnésique des ratons est plus prononcé lorsque l'alcool est consommé durant la période de la gestation. Cependant, les effets délétères induits par l'alcool durant l'allaitement maternel pourraient être masqués par le métabolisme rénal et hépatique de cette substance. Il parait en conséquence très judicieux d'évaluer les effets toxiques de la consommation d'alcool par la ratte allaitante sur les organes épurateurs des ratons allaités.

\section{CONFLIT D'INTERETS}

Les auteurs déclarent qu'ils n'ont aucun conflit d'intérêts.

\section{CONTRIBUTIONS DES AUTEURS}

KMY a proposé le thème et a élaboré le protocole de l'étude. PJDD a procédé à l'entretien des animaux, à la mesure des performances mnésiques et à la rédaction du manuscrit. NO a effectué le traitement statistique.

\section{REMERCIEMENTS}

Aux termes de ce travail, nous tenons à remercier l'Ecole Normale Supérieur (ENS) pour le cadre proposé pour la réalisation de cette étude.

\section{REFERENCES}

Alby-Averseng C. 2015. Etude clinique et génétique des anomalies du corps calleux chez le fœetus. Thèse de doctorat, Université Paris Descartes, Paris, p. 170.

Badjo P, Diboh E, Gbalou KL, Adou KF, Zunon-Kipré I, Tako NA. 2017. Effets d'une consommation prolongée de «koutoukou» (boisson alcoolique artisanale) sur la mémoire spatiale chez la souris. J. Appl. Biosci, 116: 1155711565.

DOI: https://dx.doi.org/10.4314/jab. v116i1.4

Beaulac-Baillargeon L. 2008. De la mère au nourisson. La prise d'alcool pendant la grossesse et l'allaitement. Quebec Pharmacie Press, 55(7): 13-18. DOI : https://www.professionsante.ca/files/20 09/11qp mere juillet aout08.pd

Bouamra S, Boukabous C. 2016. Evaluation de la mémoire de type épisodique chez le rat: Etude comportementale et histologique. Mémoire de Master de Biologie, Université M'hamed Bougara de Boumerdès, Boumerdès, p.49.

Cebolla AM, Cheron G, Hourez R, Bearzotto B, Dan B, Servais L. 2009. Effects of maternal alcohol consumption during breastfeeding on motor and cerebellar Purkinje cells behavior in mice. Neurosci. Lett., 455(1): 4-7. DOI : 
https://doi.org/10.1016/j.neulet.2009.03. 034

Deacon RMJ, Rawlins JNP. 2006. T-maze alternation in the rodent. Nat. Protoc., 1(1): 7-12. DOI: https://doi.org/10.1038/nprot.2006.2

Doubran PJD, Yao KM, Ouattara N, Tako AN, Seri B. 2018. Effets d'une consommation modérée d'alcool sur la progéniture de la ratte (Wistar). IJIAS, 24(4): 1690-1700.

Du Plooy CP, Malcolm-Smith S, Adnams MC, Stein DJ, Donald KA. 2016. The effects of prenatal alcohol exposure on episodic memory functioning: A systematic review. Arch Clin Neuropsychol., 31(7): 710-726. DOI: https://doi.org/10.1093/arclin/acw067

Giglia RC, Binns C. 2016. Alcohol and lactation: A systematic review. Nutr. Diet., $\quad$ 63(2): 103-116. DOI: https://doi.org:101111/j.17470080.2006.00056.x

Haastrup MB, Pottegard A, Damkier P. 2014. Alcohol and Breastfeeding. Basic Clin. Pharmacol. Toxicol., 114(2): 168-173. DOI: https://doi.org/10.1111/bcpt.12149 Ikonomidou C, Bittigau P, Ishimaru MJ, Wozniak DF, Koch C, Genz K, Price MT, Stefovska V, Hörster F, Tenkova T, Dikranian K, Olney JW. 2000. Ethanol-induced apoptotic neurodegeneration and fetal alcohol syndrome. Science, 287(5455): 10561060.

DOI: https://doi.org/10.1126/science.287.545 5.1056

Koko BK, Konan AB, Kouacou FKA, Djétouan JMK, Amonkan AK. 2019. Galactagogue Effect of Euphorbia hirta (Euphorbiaceae) aqueous leaf extract on milk production in female Wistar rats. $J$. Biosci. Med., 7(9): 51-65. DOI : https://doi.org/10.4236/jbm.2019.79006

Mennella JA, Beauchamp GK. 1991. The transfer of alcohol to human milk. Effects on flavor and the infant's behavior. N. Engl. J. Med., 325: 981985. DOI:
Organisation de coopération et de développement économiques pour les essais de produits chimiques. Essai $\mathrm{n}^{\circ}$ 426. 2007. Étude de neurotoxicité pour le développement. p. 27. DOI : https://doi.org/10.1787/20745842

Organisation de coopération et de développement économiques pour les essais de produits chimiques $\mathrm{N}^{\circ} 416$. 2001. Etude de toxicité pour la reproduction sur les deux générations. 2001. p.13. DOI : https /doi.org/10.1787/9789264070875_fr

OMS. 2017. Estimer le coût de la consommation d'alcool pendant la grossesse. Bulletin de l'Organisation Mondiale de la Santé, 9(5): 320-321. http://dx.doi.org/10.2471/BLT.17.030517

Posey D, Mozayani A. 2007. The estimation of blood alcohol concentration: Widmark revisited. Forensic. Sci. Med. Pathol., 3(1):33-39. DOI: https://doi.org/10.1385/fsmp:3:1:33

Seror E, Chapelon E, Bue M, GarnierLengline $\mathrm{H}$, Lebeaux-Legras $\mathrm{C}$, Loudenot A, Lejeune C. 2009. Alcool et grossesse, Arch Pediatr, 16 (10): 13641373.

DOI : https://doi.org/10.1016/j.arcped.2009.07 .009

De Ferron BS.2015. Alcool et mémoire : Implication de la sous-unité GluN2B et de KCC2 dans les perturbations de la plasticité synaptique bidirectionnelle dans l'hippocampe après exposition précoce à l'éthanol. Thèse de doctorat, Université de Picardie Jules Verne, Picardie, p. 242.

Zougrou NE, Tovi WMO, Blahi ANM, Kouakou K. 2019. Evaluation des effets fertilisants et embryo-toxiques de l'extrait aqueux des feuilles de Cnestis ferruginea chez le rat. Eur. Sci. J., 15(27): 231-255. DOI : https://doi.org/10.19044/esj.2019.v15n2 $7 \mathrm{p} 23$. 